\title{
BMJ Open Silent cerebral infarction and cognitive function following TAVI: an observational two-centre UK comparison of the first- generation CoreValve and second- generation Lotus valve
}

\author{
Tarique Al Musa, ${ }^{1}$ Akhlaque Uddin, ${ }^{1}$ Catherine Loveday, ${ }^{2}$ Laura E Dobson, ${ }^{1}$ \\ Mark Igra, ${ }^{3}$ Fiona Richards, ${ }^{1}$ Peter P Swoboda, ${ }^{1}$ Anvesha Singh, ${ }^{4,5}$ Pankaj Garg, ${ }^{1}$ \\ James R J Foley, ${ }^{1}$ Graham J Fent, ${ }^{1}$ Anthony J P Goddard, ${ }^{3}$ Christopher Malkin, ${ }^{3}$ \\ Sven Plein, ${ }^{1,3}$ Daniel J Blackman, ${ }^{3}$ Gerald P McCann, ${ }^{4,5}$ John P Greenwood ${ }^{1,3}$
}

To cite: Musa TA, Uddin A, Loveday C, et al. Silent cerebral infarction and cognitive function following TAVI: an observational two-centre UK comparison of the first-generation CoreValve and second-generation Lotus valve. BMJ Open 2019;9:e022329. doi:10.1136/ bmjopen-2018-022329

- Prepublication history for this paper is available online. To view these files please visit the journal online (http://dx.doi org/10.1136/bmjopen-2018022329).

Received 14 February 2018 Revised 5 August 2018 Accepted 26 November 2018

Check for updates

(C) Author(s) (or their employer(s)) 2019. Re-use permitted under CC BY-NC. No commercial re-use. See rights and permissions. Published by BMJ.

For numbered affiliations see end of article.

Correspondence to Dr John P Greenwood; j.greenwood@leeds.ac.uk

\section{ABSTRACT}

Objective To compare the incidence of silent cerebral infarction and impact on cognitive function following transcatheter aortic valve implantation (TAVI) with the firstgeneration CoreValve (Medtronic, Minneapolis, Minnesota, USA) and second-generation Lotus valve (Boston Scientific, Natick Massachusetts, USA).

Design A prospective observational study comprising a 1.5 T cerebral MRI scan, performed preoperatively and immediately following TAVI, and neurocognitive assessments performed at baseline, 30 days and 1 year follow-up.

Setting University hospitals of Leeds and Leicester, UK. Patients 66 ( $80.6 \pm 8.0$ years, $47 \%$ male) patients with high-risk severe symptomatic aortic stenosis recruited between April 2012 and May 2015.

Main outcome measures Incidence of new cerebral microinfarction and objective decline in neurocognitive performance.

Results All underwent cerebral MRI at baseline and immediately following TAVI, and 49 (25 Lotus, 24

CoreValve) completed neurocognitive assessments at baseline, 30 days and 1 year. There was a significantly greater incidence of new cerebral microinfarction observed following the Lotus TAVI (23 (79\%) vs $22(59 \%), p=0.025)$ with a greater number of new infarcts per patient (median 3.5 (IQR 7.0) vs 2.0 (IQR 3.0), $p=0.002$ ). The mean volume of infarcted cerebral tissue per patient was equivalent following the two prostheses $(p=0.166)$. More patients suffered new anterior $(14(48 \%)$ vs $2(5 \%), p=0.001)$ and vertebrobasilar ( $15(52 \%)$ vs $7(19 \%), p=0.005)$ lesions following Lotus. Lotus was associated with a decline in verbal memory and psychomotor speed at 30 days. However, performance longitudinally at 1 year was preserved in all neurocognitive domains.

Conclusions There was a higher incidence of silent cerebral microinfarction and a greater number of lesions per patient following Lotus compared with CoreValve. However, there was no objective decline in neurocognitive function discernible at 1 year following TAVI with either prosthesis.

\section{Strengths and limitations of this study}

- Contemporary study comprising both a novel transcatheter aortic valve implantation (TAVI) design technology and an established device.

- Atwo-centre study combining both diffusion-weighted MRI and cognitive assessments to comprehensively assess cerebral injury following TAVI.

Mid-term follow-up to 1 year.

- Not randomised.

- Higher field strength MRI available for more sensitive detection of cerebral embolic infarction.

\section{INTRODUCTION}

Transcatheter aortic valve implantation (TAVI) is advocated by both European ${ }^{1}$ and $\mathrm{US}^{2}$ guidance in patients with symptomatic aortic stenosis (AS) deemed inoperable or with too high a predicted postoperative mortality. TAVI is cost-effective, ${ }^{3}$ significantly improves quality of life ${ }^{4}$ and, as recent trial data suggest, non-inferior to surgery in intermediate-risk patients, ${ }^{5}$ potentially expanding its application to a broader population.

However, since the landmark Placement of Aortic Transcatheter Valve Trial (PARTNER) in $2010,{ }^{6}$ the inherent risk of disabling stroke associated with TAVI has been well recognised. Despite 10 years of refinement in transcatheter technology and delivery techniques, stroke rates remain similar. ${ }^{7}$ Importantly, TAVI is also associated with a high incidence (up to 84\%) of silent cerebral embolism as detected by diffusion-weighted MRI (DW-MRI) ${ }^{8-10}$ more frequent than that following aortic valve surgery. ${ }^{11}$

The association of new DW-MRI lesions post-TAVI with cognitive decline is an issue 
currently under intense scrutiny, as it has long been recognised that silent cerebral infarcts can more than double the risk of dementia. ${ }^{12}$ Indeed the concept of postoperative cognitive dysfunction and its relationship with DW-MRI has been described following valvular surgery, bypass grafting and left heart catheterisation. ${ }^{13}$

The Boston Scientific Lotus valve is a novel TAVI iteration that incorporates a number of features specifically designed to improve on first-generation devices. ${ }^{14}$ As such it exhibits superior deployment success rates when compared with the older Medtronic CoreValve. ${ }^{1516}$ However, the key concern of silent cerebral injury following implantation and cognitive decline remains unanswered. We sought to characterise the extent of new silent cerebral infarction using DW-MRI, and investigate longitudinally cognitive function following TAVI with the first-generation self-expanding Medtronic CoreValve and the second-generation mechanically-expanded Boston Scientific Lotus valve.

\section{METHODS}

\section{Patient selection}

This study prospectively recruited 74 patients with severe degenerative aortic stenosis (AS) from two large UK tertiary cardiothoracic centres (Leeds and Leicester) who were referred for TAVI between April 2012 and May 2015. Severe AS was classified by echocardiography as an aortic valve area of $\leq 1.0 \mathrm{~cm}^{2}$ or peak velocity $>4 \mathrm{~m} / \mathrm{s}$. The decision for TAVI in all cases was taken by a multidisciplinary heart team in accordance with international guidance ${ }^{17}$ Exclusion criteria included any contraindication to MRI or pre-existing severe cognitive impairment (a Mini-Mental State Examination (MMSE) Score $<10$ ). Any patient deemed to exhibit new focal neurological dysfunction consistent with clinical stroke post-TAVI was also excluded. The study complied with the Declaration of Helsinki and all patients provided written informed consent.

\section{Transcatheter aortic valve implantation}

TAVI was performed using either a first-generation CoreValve system (Medtronic, Minneapolis, Minnesota, USA) or the Lotus aortic valve system (Boston Scientific Corporation, Natick, Massachusetts, USA) employing standard techniques as previously described for both vendors. ${ }^{18} 19$ Multidetector CT was employed to assist annular sizing and assess aortic calcification prior to TAVI. Percutaneous femoral artery access was the default approach. Balloon valvuloplasty, rapid ventricular pacing and postdilatation (in the case of CoreValve) were employed at the discretion of the operator. All patients received weight-adjusted unfractionated heparin to maintain an activated clotting time $>200$ s and were treated with dual antiplatelet therapy (aspirin $75 \mathrm{mg}$ and clopidogrel $75 \mathrm{mg}$ ) for a minimum of 3 months. None of the TAVI cases involved the use of a cerebral protection device.

\section{Neurocognitive assessment}

The neurocognitive test battery was performed by trained assessors (medical/nursing) in a quiet comfortable environment at three Valve Academic Research Consortium recommended time points (baseline, 30 days and 12 months). ${ }^{20}$ Training of assessors and performance validation was undertaken by an experienced neuropsychologist (CL). Follow-up assessment was conducted at the patient's home or the hospital. Total assessment time ranged between $60 \mathrm{~min}$ and $90 \mathrm{~min}$ with appropriate rest periods. Baseline characteristics, patient handedness and years of education were recorded. The National Adult Reading Test (NART) was used to calculate the full scale intelligent quotient $(123.2-(1.029 \times$ NART error score $))$. A broad battery of previously validated neurocognitive assessments was undertaken at the designated time points ${ }^{21}$ and included evaluation of: cognitive reasoning: using MMSE, verbal memory: using the Hopkins Verbal Learning Test (HVLT), executive function: using the Letter Number Sequencing task, psychomotor speed: using the Trail Making Tests (TMTs) A and B, perceptual and visual memory: using the Digit-Symbol Substitution Test, and fine motor coordination and speed: using the Grooved Pegboard Test (Model 32025, Lafayette Instruments, Lafayette, Indiana, USA). Cognitive decline was defined as a reduction of the score by $1 \mathrm{SD}$ of the baseline score for all tests. ${ }^{22-24}$

\section{Cerebral MRI}

Cerebral MRI was conducted preprocedure and postprocedure (within 7 days) using identical imaging protocols. MRI was performed on the same $1.5 \mathrm{~T}$ system for all serial scans for any individual patient (Intera, Phillips Healthcare, Best, The Netherlands or Avanto, Siemens Medical Systems, Erlangen, Germany). The imaging protocol consisted of T2-weighted fast field echo, T2 turbo field echo and diffusion-weighted imaging (DWI) (22 slices, $5 \mathrm{~mm}$ thick, $1 \mathrm{~mm}$ gap, Field of View (FOV) 350, Rectangular Field of View (RFOV) 100. Each scan was independently assessed by two experienced neuroradiologists (AJPG, MI), blinded to all clinical/procedural details. Cerebral embolism or microinfarction was defined as a new restricted diffusion lesion on DWI. New cerebral microinfarcts were localised to one hemisphere and vascular territory. Infarct diameter was used to categorise patients into small or large lesion subgroups $(<5 \mathrm{~mm}$ or $>5 \mathrm{~mm}$; in the case of multiple lesions the largest lesion was used to determine status). The total infarct volume $(\mathrm{mL})$ was measured off-line using standard postprocessing software (QMass V.7.2, Medis, The Netherlands) as previously published. ${ }^{1011}$

\section{Statistical analysis}

Data were tested for normality using the Shapiro-Wilk test. Continuous variables were expressed as mean \pm SD or median (Q2-Q3 or IQR) and were tested for differences by means of a two-sided, unpaired Student's t-test (for comparison between groups) or a two-sided, paired Student's t-test (for intragroup comparison). 
Table 1 Patient characteristics in those with 6-month follow-up

\begin{tabular}{llll}
\hline & $\begin{array}{l}\text { Lotus } \\
(\mathbf{n = 2 9 )}\end{array}$ & $\begin{array}{l}\text { CoreValve } \\
(\mathbf{n = 3 7 )}\end{array}$ & P value $^{*}$ \\
\hline Age (years) & $79.8 \pm 8.9$ & $81.2 \pm 7.2$ & 0.459 \\
\hline Male, $\mathrm{n}(\%)$ & $14(48)$ & $17(46)$ & 0.851 \\
\hline EuroSCORE II & $4.13 \pm 3.30$ & $5.55 \pm 3.79$ & 0.115 \\
\hline STS Score (\%) & $4.04 \pm 2.59$ & $5.28 \pm 3.41$ & 0.109 \\
\hline BMI (kg/m $\left.{ }^{2}\right)$ & $28.5 \pm 5.2$ & $27.2 \pm 5.4$ & 0.323 \\
\hline Hypertension, $\mathrm{n}(\%)$ & $11(38)$ & $20(54)$ & 0.157 \\
\hline Diabetes, $\mathrm{n}(\%)$ & $5(17)$ & $4(11)$ & 0.450 \\
\hline Hyperlipidaemia, $\mathrm{n}(\%)$ & $17(59)$ & $18(49)$ & 0.488 \\
\hline Atrial fibrillation, $\mathrm{n}(\%)$ & $5(17)$ & $9(24)$ & 0.449 \\
\hline Previous MI, $\mathrm{n}(\%)$ & $7(24)$ & $2(5)$ & $\mathbf{0 . 0 2 6}$ \\
\hline Previous PCl, $\mathrm{n}(\%)$ & $9(31)$ & $10(27)$ & 0.774 \\
\hline Previous CABG, $\mathrm{n}(\%)$ & $5(17)$ & $8(22)$ & 0.618 \\
\hline Previous stroke, $\mathrm{n}(\%)$ & $7(24)$ & $6(16)$ & 0.454 \\
\hline $\begin{array}{l}\text { Peripheral vascular } \\
\text { disease, } \mathrm{n}(\%)\end{array}$ & $4(14)$ & $5(14)$ & 0.991 \\
\hline eGFR (ml/min/1.73 m²) & $69 \pm 19$ & $64 \pm 17$ & 0.342 \\
\hline
\end{tabular}

Values are mean \pm SD or $\mathrm{n}(\%)$.

${ }^{*} \mathrm{P}$ value for comparison between procedure types.

BMI, body mass index; CABG, coronary artery bypass grafting; eGFR, estimated glomerular filtration rate; EuroSCORE, European System for Cardiac Operative Risk Evaluation; MI, myocardial infarction; $\mathrm{PCl}$, percutaneous coronary intervention; STS, Society of Thoracic Surgeons.

Non-parametrical testing (Mann-Whitney test) was performed where indicated. Categorical variables were given as frequencies and percentages and compared by $\chi^{2}$ statistics or Fisher's exact test. The PASW software package (V.21.0 SPSS, IBM, Chicago, Illinois, USA) was used for all statistical analyses and a two-sided significance level of $\mathrm{p}<0.05$ was considered statistically significant. The sample size required to detect cognitive decline (defined as a reduction in score by $1 \mathrm{SD}$ ) was estimated using IBM SPSS sample power V.3, and using normative data for the test to have $80 \%$ power $(\alpha 0.05)$, a minimum of 17 patients was required in each group.

\section{RESULTS}

\section{Patient population}

A total of 66 patients (37 Medtronic CoreValve and 29 Boston Lotus) underwent both the preoperative (median 1 day preprocedure, IQR 14 days) and immediate postTAVI MRI scans (median 4 days, IQR 4 days). The baseline characteristics of these groups are shown in table 1. Fortynine of these (24 CoreValve and 25 Lotus) completed serial neurocognitive assessments up to 12 months. Reasons for non-completion were varied reflecting an elderly frail population (figure 1).

\section{Patient involvement}

Patients were not involved with the study design, recruitment, conduct or interpretation of results obtained. Outcome measures were objectively measured and not based on patient experience.

\section{Procedural data}

TAVI was successful in all cases. Catheterisation data for the TAVI implant procedures are summarised in table 2. Of the 29 Lotus implants, 7 (24\%) involved device repositioning. Of the CoreValve implants, there were three instances of embolisation and the requirement of a second valve in three cases (two of whom were due to embolisation).

\section{Cerebral MRI}

Typical cerebral DWI images at baseline and immediately after TAVI are depicted in figure 2A,B, respectively.

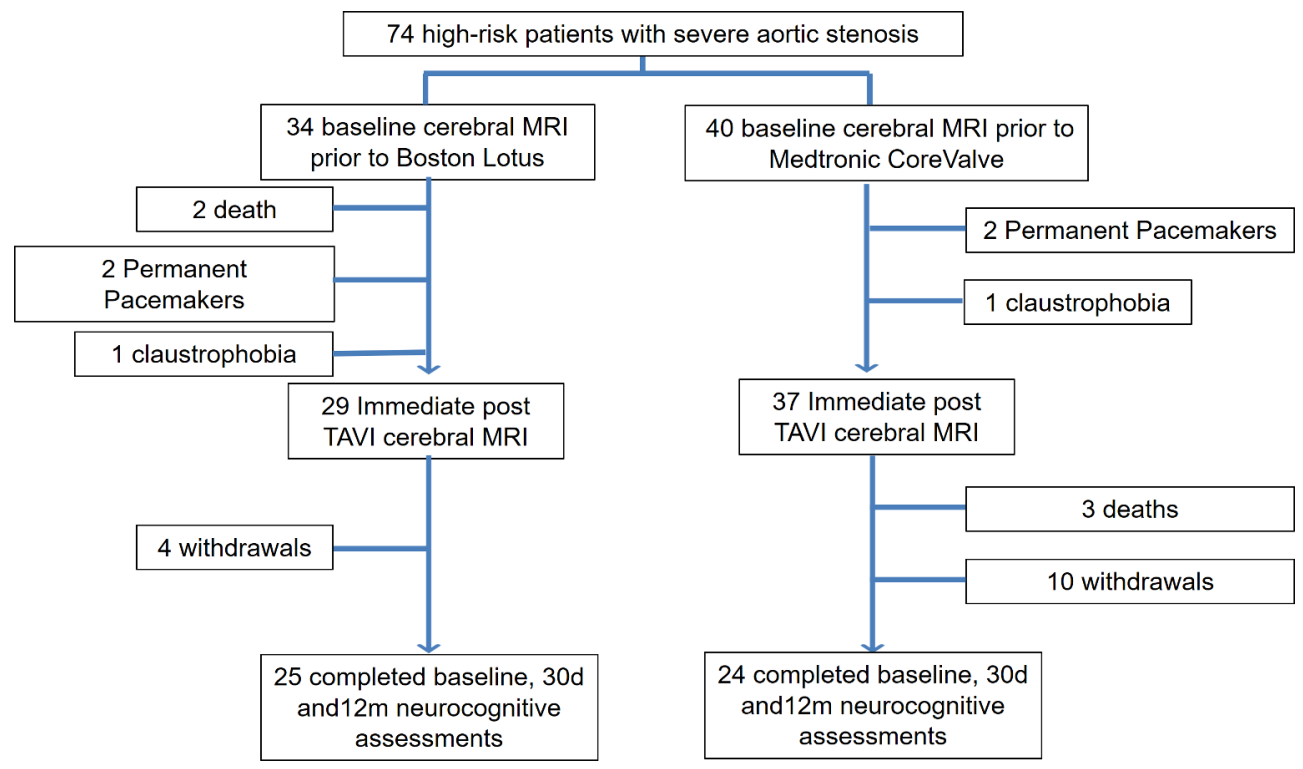

Figure 1 Study profile. TAVI, transcatheter aortic valve implantation. 
Table 2 Catheterisation data for TAVI implant procedures

\begin{tabular}{|c|c|c|c|}
\hline & Lotus $(n=29)$ & CoreValve $(n=37)$ & P value* \\
\hline TAVI size $(n(\%))$ & $\begin{array}{l}23 \mathrm{~mm}(7(24 \%)) \\
25 \mathrm{~mm}(8(28 \%)) \\
27 \mathrm{~mm}(14(48 \%))\end{array}$ & $\begin{array}{l}23 \mathrm{~mm}(4(11 \%)) \\
26 \mathrm{~mm}(8(22 \%)) \\
29 \mathrm{~mm}(18(49 \%)) \\
31 \mathrm{~mm}(7(19 \%))\end{array}$ & - \\
\hline Femoral route, n (\%) & $29(100)$ & $27(73)$ & 0.081 \\
\hline Sheath size (French) & $\begin{array}{l}18(28 \%) \\
20(52 \%) \\
22(20 \%)\end{array}$ & $18(100 \%)$ & \\
\hline Pullback PG (mm Hg) & $56 \pm 27$ & $43 \pm 19$ & 0.023 \\
\hline Fluoroscopy time (min) & $29 \pm 8$ & $18 \pm 11$ & 0.001 \\
\hline Procedure time (min) & $159 \pm 42$ & $143 \pm 50$ & 0.194 \\
\hline Contrast (ml) & $120 \pm 43$ & $134 \pm 43$ & 0.212 \\
\hline Predilatation BAV, n (\%) & $25(86)$ & $28(77)$ & 0.286 \\
\hline Postdilatation, $\mathrm{n}(\%)$ & $0(0)$ & $5(14)$ & 0.039 \\
\hline TAVI repositioned, n (\%) & $7(24)$ & $0(0)$ & 0.002 \\
\hline TAVI embolisation, $\mathrm{n}(\%)$ & $0(0)$ & $3(8)$ & 0.117 \\
\hline Need for second TAVI, n (\%) & $0(0)$ & $3(8)$ & 0.117 \\
\hline
\end{tabular}

${ }^{*}$ Independent samples t-test

PG, pressure gradient; BAV, balloon aortic valvuloplasty; TAVI, transcatheter aortic valve implantation.

Baseline. At baseline, $5(17 \%)$ of the Lotus patients and $10(27 \%)$ of the CoreValve patients had evidence of pre-existing established stroke $(\mathrm{p}=0.346)$ with equivalent lesion volume $(\mathrm{p}=0.529)$. There was also evidence of recent microinfarction on cerebral DWI in two $(7 \%)$ Lotus and four $(11 \%)$ CoreValve patients $(\mathrm{p}=0.583)$. A greater proportion of patients undergoing Lotus TAVI had evidence of periventricular ischaemia compared with those undergoing CoreValve TAVI (19 (66\%) vs $14(38 \%), \mathrm{p}=0.026)$.

Postprocedure:Table 3 summarises the findings of DWI following TAVI. There was a significantly greater incidence of new microinfarction observed following Lotus TAVI with a greater number of new infarcts per patient. These were predominantly of small size, with a comparable mean

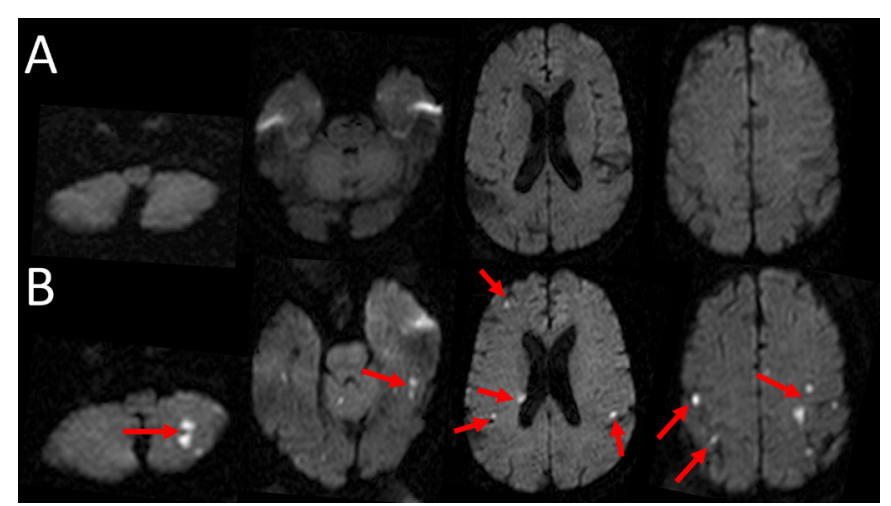

Figure 2 Diffusion-weighted MRI of the brain examining silent injury with transcatheter aortic valve implantation (TAVI). Cerebral images, including the brainstem and cerebellum before (A) and after (B) the TAVI procedure. Multiple new cerebral infarctions were seen, some of which are highlighted by the red arrows. infarcted cerebral tissue volume per patient with both TAVI designs. More patients suffered new anterior and vertebrobasilar lesions following Lotus than CoreValve. The number of new lesions following Lotus repositioning $(n=7)$ and non-repositioning $(\mathrm{n}=22)$ did not differ (1.0 (IQR 8.0) vs 3.0 (IQR 3.75), $\mathrm{p}=0.438$ ) and Lotus repositioning did not correlate with the number of new lesions $(\mathrm{p}=0.435)$. For the CoreValve group, the three patients in whom embolisation occurred had significantly greater frequency of new lesions postprocedure (4.0 (IQR 0.0) vs 1.0 (IQR 2.0), p=0.011), but no difference was seen in the three requiring a second valve (4.0 (IQR 0.0) vs 1.0 (IQR 2.0), p=0.148). Typical findings of new cerebral infarctions detected by DW-MRI post-TAVI are exemplified in figure 2.

\section{Baseline education and intelligence}

The vast majority of patients was right-handed (CoreValve: $21(88 \%)$ vs Lotus: $24(96 \%), \mathrm{p}=0.234)$ with an equivalent reporting of disability on the Modified Rankin Scale (CoreValve: $1.9 \pm 1.1$ vs Lotus: $2.0 \pm 0.7, \mathrm{p}=0.929$ ). The CoreValve and Lotus patients were similar with respect to years of education (10.9 \pm 1.9 years vs $11.6 \pm 2.5$ years, $\mathrm{p}=0.279)$ and full scale IQ $(106 \pm 12$ vs $102 \pm 15, \mathrm{p}=0.313)$. Global cognition measured using MMSE was also equivalent (28.8 \pm 1.8 vs $28.6 \pm 1.6$, $\mathrm{p}=0.279$ ) with only one patient from the Lotus group indicating cognitive impairment (defined as MMSE $<24$ ). ${ }^{25}$

\section{Effect of TAVI design on absolute neurocognitive function} scores

Neurocognitive performance, prior to and following Lotus and CoreValve TAVI, are shown in table 4. For the vast majority of domains, including global cognition, psychomotor speed, executive function and fine motor 
Table 3 Comparison of MRI DWI following Boston Lotus and Medtronic CoreValve

\begin{tabular}{|c|c|c|c|}
\hline & Lotus $(n=29)$ & CoreValve $(n=37)$ & $P$ value \\
\hline Incidence of new microinfarction (n(\%)) & 23(79) & $22(59)$ & 0.025 \\
\hline Number of new microinfarcts per patient & 3.5 (IQR 7.0) & 2.0 (IQR 3.0) & 0.002 \\
\hline Number of new small lesions $(<5 \mathrm{~mm})(\mathrm{n}(\%))$ & $124(78)$ & $41(72)$ & 0.005 \\
\hline Number of new large lesions ( $\geq 5 \mathrm{~mm})(\mathrm{n}(\%))$ & $34(22)$ & $16(28)$ & 0.036 \\
\hline Number of patients with new MCA lesions $(n(\%))$ & 20(69) & $19(51)$ & 0.149 \\
\hline Number of patients with new PCA lesions (n(\%)) & 10(34) & $6(16)$ & 0.086 \\
\hline Number of patients with new VBA lesions (n(\%)) & 15(52) & $7(19)$ & 0.005 \\
\hline
\end{tabular}

ACA, anterior cerebral artery; DWI, diffusion-weighted imaging; MCA, middle cerebral artery; PCA, posterior cerebral artery; VBA, vertebrobasilar artery

Statistically significant $P$ values $<0.05$ are shown in bold.

coordination, no change in test scores was observed at 30 days or 12 months following TAVI, with either the Lotus or CoreValve prostheses.

HVLT was used to test mnemonic function and verbal memory, and total learning scores remained unchanged over time for both CoreValve and Lotus. However, delayed recall scores and discrimination index were significantly lower at 30 days following Lotus (table 4), with 12-month scores returning to baseline level (delayed recall: 7.6 \pm 3.0 vs $9.0 \pm 6.7, \mathrm{p}=0.320$, discrimination index: $10.2 \pm 1.5$ vs $10.1 \pm 2.4, \mathrm{p}=0.867$ ), with no change observed following CoreValve.

TMT assesses executive function and psychomotor speed, with TMT A preceding the more challenging

Table 4 Summary of neurocognitive test scores at baseline, 30 days and 12 months

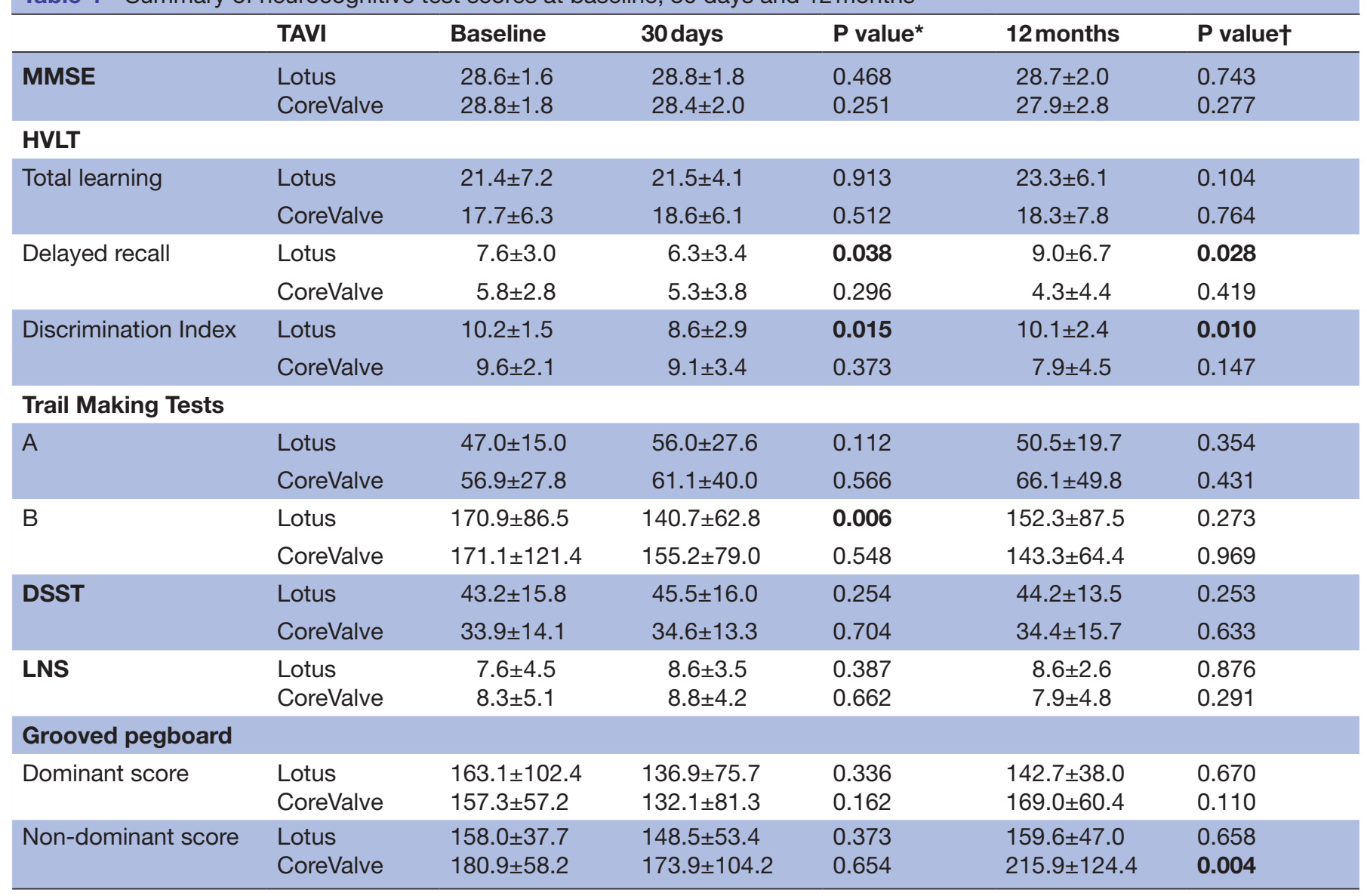

Mean \pm SD.

${ }^{*}$ Comparing values at baseline and 30 days, same TAVI design, paired samples t-test.

†Comparing values at 30 days and 12 months, same TAVI design, paired samples t-test.

DSST, Digit-Symbol Substitution Test; HVLT, Hopkins Verbal Learning Test; LNS, Letter Number Sequencing; MMSE, Mini-Mental State

Examination; TAVI, transcatheter aortic valve implantation. 
Table 5 Within-group comparison of mean change in cognitive domain over 12 months in patients with and without diffusionweighted (DW) MRI lesions

\begin{tabular}{|c|c|c|c|c|c|c|}
\hline & \multicolumn{3}{|c|}{ Boston Lotus $(n=25)$} & \multicolumn{3}{|c|}{ Medtronic CoreValve $(n=24)$} \\
\hline & DWI -ve $(n=6)$ & DWI +ve (n=19) & $P$ value & DWI -ve (n=11) & DWI +ve (n=13) & $P$ value \\
\hline MMSE & $1.2 \pm 0.8$ & $-0.4 \pm 1.9$ & 0.025 & $-0.9 \pm 2.5$ & $-0.7 \pm 2.6$ & 0.840 \\
\hline HVLT learning & $4.2 \pm 5.2$ & $1.2 \pm 7.3$ & 0.454 & $1.1 \pm 3.9$ & $1.0 \pm 9.1$ & 0.691 \\
\hline HVLT delayed & $6.3 \pm 12.9$ & $-0.1 \pm 3.3$ & 0.177 & $-0.2 \pm 2.5$ & $-1.1 \pm 3.1$ & 0.456 \\
\hline HVLT discrimination & $0.0 \pm 1.5$ & $-0.1 \pm 2.6$ & 0.733 & $0.8 \pm 2.3$ & $-2.3 \pm 3.5$ & 0.019 \\
\hline TMT A & $15.5 \pm 20.8$ & $-0.1 \pm 15.3$ & 0.059 & $18.9 \pm 64.9$ & $5.0 \pm 14.7$ & 0.562 \\
\hline TMT B & $-13.0 \pm 88.9$ & $-18.3 \pm 61.4$ & 0.870 & $19.6 \pm 61.6$ & $-1.4 \pm 64.4$ & 0.454 \\
\hline DSST & $4.0 \pm 10.0$ & $-0.4 \pm 9.8$ & 0.346 & $-0.1 \pm 6.7$ & $5.0 \pm 13.7$ & 0.370 \\
\hline LNS & $2.4 \pm 3.0$ & $0.7 \pm 5.1$ & 0.534 & $-0.5 \pm 5.0$ & $0.8 \pm 3.6$ & 0.474 \\
\hline GPBT dominant & $-8.2 \pm 31.5$ & $-24.5 \pm 108.0$ & 0.721 & $0.5 \pm 37.6$ & $22.6 \pm 37.7$ & 0.186 \\
\hline GPBT non-dominant & $-26.0 \pm 83.7$ & $-3.2 \pm 51.3$ & 0.914 & $43.0 \pm 81.3$ & $20.8 \pm 66.8$ & 0.235 \\
\hline
\end{tabular}

Mean \pm SD.

DSST, Digit-Symbol Substitution Test; DWI, diffusion-weighted imaging; GPBT, Grooved Pegboard Test; HVLT, Hopkins Verbal Learning Test; LNS, Letter Number Sequencing; MMSE, Mini-Mental State Examination; TMT, Trail Making Test.

TMT B. TMT B scores were significantly lower at 30 days following Lotus (table 4), with 12-month scores again returning to the baseline level $(170.9 \pm 86.5$ vs $152.3 \pm 87.5$, $\mathrm{p}=0.215$ ), and no change observed following CoreValve at any time point.

\section{Presence or absence of DW-MRI lesions}

At the 1-year follow-up, for each TAVI prosthesis we sought to compare individuals with and without DW-MRI lesions with respect to neurocognitive performance (table 5). Of

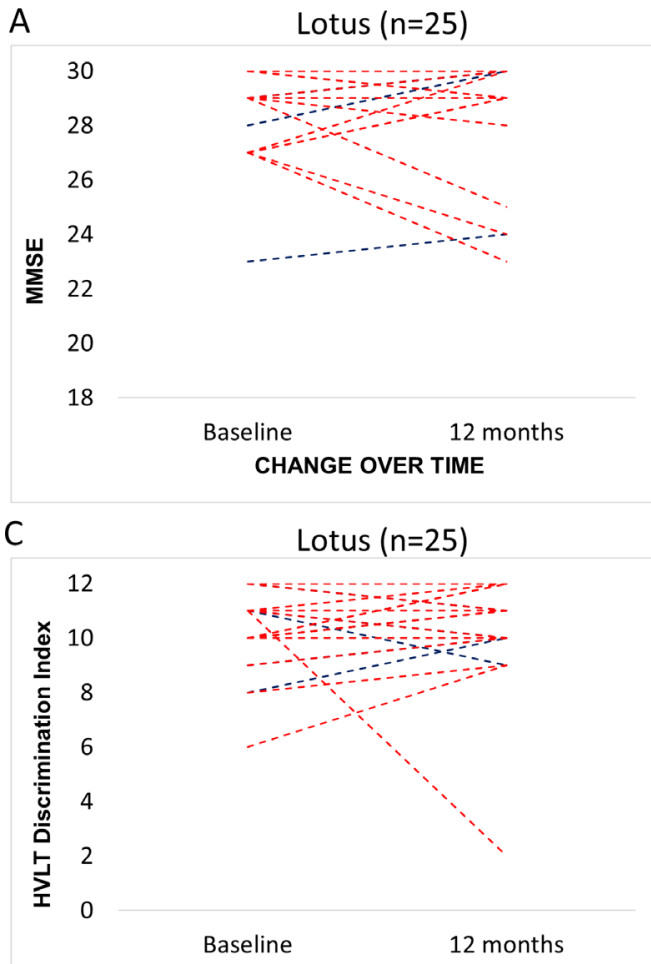

CHANGE OVER TIME the 25 Lotus patients that completed 12-month assessments, $19(76 \%)$ exhibited new DW-MRI lesions postTAVI, and of the 24 CoreValve patients, $13(54 \%)$ were DW-MRI positive $(\mathrm{p}=0.140)$. Lotus patients with DW-MRI lesions exhibited an overall decline in global cognition (MMSE) at 12 months compared with those without (figure 3A,B). CoreValve patients with DW-MRI lesions had a significantly lower HVLT discrimination index at 12 months compared with those without (figure 3C,D).

B

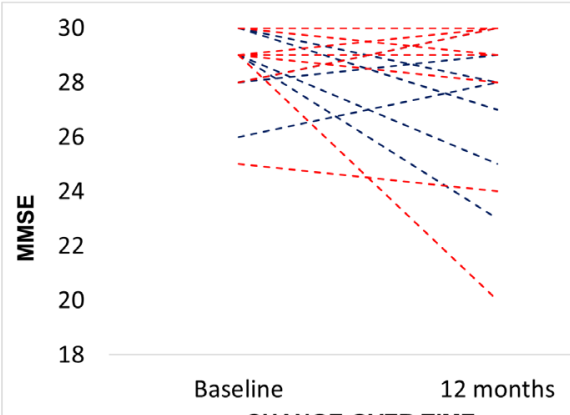

CHANGE OVER TIME

D

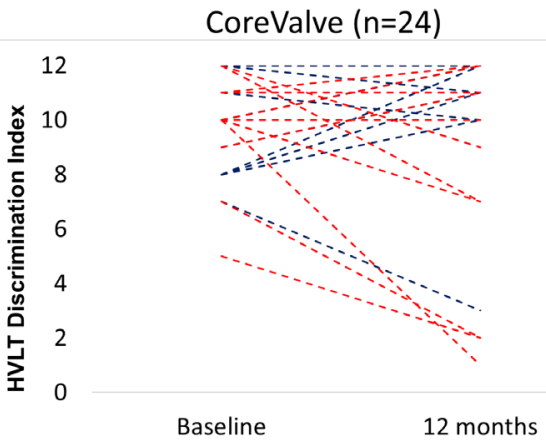

CHANGE OVER TIME

Figure 3 Line graph depicting change in Mini-Mental State Examination (MMSE) (A and B) and Hopkins Verbal Learning Test (HVLT) Discrimination Index (C and D) over time following Lotus and CoreValve. (Red lines indicate patients with diffusionweighted (DW) MRI microinfarcts, blue lines those without). 
For all of the remaining neurocognitive domains, no difference in performance at 1 year was noted between patients with and without DW-MRI lesions for either TAVI prosthesis.

\section{DISCUSSION}

This two-centre study is the first to use DW-MRI and cognitive assessments to comprehensively compare two first-generation and second-generation TAVI devices. This study provides several new insights: (1) There was a high occurrence of silent cerebral embolism during TAVI affecting both first-generation and second-generation prosthesis designs (with $66 \%$ of the total study population exhibiting new DW-MRI lesions post-TAVI). (2) The Lotus valve exhibited a significantly higher incidence of new DW-MRI microinfarcts, with more than twice the number of lesions per patient than observed following CoreValve. (3) DW-MRI lesions are of similar size, but more frequently observed in the anterior cerebral and vertebrobasilar territories following Lotus compared with CoreValve. (4) Despite a higher DW-MRI burden and an initial deterioration in verbal memory following Lotus, TAVI does not appear detrimental to mid-term neurocognitive function, with 12-month scores in all domains being equivalent to baseline following both Lotus and CoreValve. (5) For the majority of domains, including executive function, psychomotor speed, perceptual and visual memory, and fine motor coordination and speed, the presence of DW-MRI lesions did not influence neurocognitive function at 1 year for either valve. This latter point is consistent with previous work that demonstrated preserved 2-year cognitive performance in an unselected TAVI population, irrespective of DW-MRI status. ${ }^{23}$

Cognitive decline after cardiac surgery is associated with increased morbidity and mortality ${ }^{26}$ and has significant social and economic implications. ${ }^{23}$ TAVI has revolutionised the management of high-risk patients with symptomatic aortic stenosis worldwide. However, determining whether the high incidence of silent DW-MRI lesions seen post-TAVI predisposes to cognitive decline, and whether TAVI design has any impact on this, remains a crucial prerequisite to its more widespread use. Despite its advanced design, our study suggests that the use of the second-generation Lotus valve carries a higher risk of silent cerebral infarction, but without an objectively discernible decline in neurocognitive function at 1 year, comparable to the CoreValve prosthesis.

The multiple and diffusely distributed silent cerebral lesions detected by DW-MRI post-TAVI are in keeping with an embolic aetiology. We have previously shown severity of aortic arch atheroma is an independent risk factor for the development of new cerebral infarcts following TAVI. ${ }^{10}$ Transcranial Doppler studies, performed during TAVI, have indicated balloon valvuloplasty, prosthesis positioning and implantation as particular moments for cerebral embolisation, ${ }^{27}$ suggesting that manipulation of the native aortic valve is also an important source.
Oversizing has been associated with tissue embolisation on histopathological analysis ${ }^{28}$ and is recommended to a degree with first-generation designs in order to prevent paravalvular regurgitation. The higher cover index and greater displacement forces on degenerate native leaflets might consequently be expected to precipitate a greater degree of tissue dehiscence and embolisation. Balloon postdilatation has also been demonstrated to significantly predict acute neurological events. ${ }^{7}$ Despite the larger valve size, greater frequency of valve embolisation and use of postdilatation, new DW-MRI lesions were less frequently seen following CoreValve. The Lotus valve is fully repositionable, reflecting the longer fluoroscopy times observed with this prosthesis. However, our data suggest that repositioning per se was not a significant contributor to the frequency of new DW-MRI lesions observed. The Lotus delivery system typically requires $20 \mathrm{~F}$ or wider sheaths, and thus greater endothelial disruption during manipulation around the aortic arch may underlie the higher incidence of cerebral microinfarction seen after Lotus TAVI. Future studies are required to determine whether these findings may translate into variation in the incidence of clinical stroke.

We and others have previously demonstrated that majority of DW-MRI lesions immediately post-TAVI appear to resolve by 6 months. ${ }^{11}$ However, this resolution does not necessarily indicate tissue normalisation. The insensitivity of lower field strength imaging has been suggested as one explanation. ${ }^{13}$ Furthermore, rat models of cerebral ischaemic insult confirm histological neuronal damage despite DW-MRI resolution, cautioning the use of imaging alone to assess ischaemic injury. ${ }^{29}$ We therefore sought to compliment cerebral MRI with a battery of well-validated neurocognitive assessment tools.

There is a large body of evidence to indicate that silent cerebral injury heralds adverse cognitive consequences. They are associated with an increased risk of mild cognitive impairment, ${ }^{30}$ and may double the risk of dementia (most commonly Alzheimer's disease), with a steeper rate of cognitive decline observed the greater the number of infarctions. ${ }^{12}$ It is feasible that the decline in verbal memory seen at 30 days following Lotus, which was not observed following CoreValve, is a reflection of the associated higher burden of DW-MRI lesions.

To date, few studies have combined DW-MRI and cognitive assessment following TAVI with CoreValve or Edwards-Sapien. The largest involved 111 subjects ${ }^{23}$ with the other three averaging 40 patients. ${ }^{82} 31$ No decline in any cognitive domain was observed despite the occurrence of diffuse microinfarcts affecting both cerebral hemispheres, ${ }^{22}$ and patients with and without DW-MRI lesions performed equally well. ${ }^{23}$ These findings are consistent with our study. Our observed high incidence of new DW-MRI lesions post-TAVI appeared to lack clinical sequelae when cognition was objectively assessed at 
12 months, and this was the case following both first-generation and second-generation devices.

This study is noteworthy in the context of contemporary TAVI trials. Results from the PARTNER 2 Trial have suggested that transfemoral TAVI results in a lower rate of death or disabling stroke in intermediate-risk patients, with an average age similar to this study of 81.5 years, when compared with surgery. ${ }^{32}$ Younger patients would expectedly exhibit lower burdens of aortic atheroma and thus fewer silent DW-MRI lesions, irrespective of TAVI design. In the recent Claret Embolic Protection and TAVI $\left(\right.$ CLEAN-TAVI) ${ }^{33}$ and SENTINEL ${ }^{34}$ trials, cerebral protection reduced new ischaemic cerebral lesions. However, Lotus patients were notably absent from both studies. While pre-existing lesion volume predicted future cerebral lesion volume,${ }^{34}$ the baseline and post-TAVR volume of established DWI lesions in our study of both CoreValve and Lotus were equivalent. Our work suggests that devices to reduce lesion frequency may be a particularly pertinent adjunct to Lotus TAVI and potentially offset the decline in verbal memory and psychomotor speed we observed at 30 days.

While our work indicates DW-MRI lesions do not seem to affect mid-term neurocognitive function, caution is required not to dismiss DW-MRI lesions as entirely innocuous, particularly given even small lesions tripled the risk of stroke-related death in healthy subjects aged $50-73$ years over 14 years of follow-up..$^{35}$ Longer follow-up is required to clinically appreciate the natural history of cerebral injury incurred during TAVI.

\section{Limitations}

Patients were not randomised to CoreValve or Lotus and hence the study is vulnerable to selection bias. Furthermore, we have not directly compared cognitive performance from our two TAVI groups with that of healthy octogenarians, or patients managed conservatively.

There was attrition in patients completing the study protocol, with 5 patients failing to complete postintervention imaging and 17 patients with imaging data failing to complete 12-month neurocognitive assessments. Fourteen patients (19\%) withdrew from the study and this was often due to deteriorating health or transfer into longterm nursing care There is hence an inherent risk of bias as potentially those with most marked decline in cognitive function might have been excluded. Furthermore, our finally analysed patient group sizes confer limited power to report 'no difference' in baseline variables raising the possibility of type 1 and type 2 errors, and transfer bias influencing our final group comparisons.

The CoreValve has been clinically in use for much longer than the Lotus valve. Therefore, the greater degree of new DWI lesions seen in the Lotus group may partly reflect an operator 'learning curve' during which experience and fluency in use of Lotus delivery equipment was refined.

Presently, there is no internationally accepted definition of cognitive decline following cardiac procedures, with the potential for variation between studies. We have used a comprehensive battery of validated tests that cover a wide variety of important higher neurocognitive faculties, but these may potentially be insensitive to change and lack validation in the context of TAVI.

Patients in atrial fibrillation on formal anticoagulation had their warfarin withheld prior to their TAVI procedure, with full dose heparin during the TAVI implant and recommencement of warfarin on the evening of the procedure. However, it is not possible to exclude cardiac thrombus associated with atrial fibrillation as a potential contributor to microinfarction.

Our work employed 1.5 T field strength imaging, which is the case for the majority of similar published studies. However, the use of higher field strengths may have increased detection of microinfarction and characterise more accurately the burden associated with different TAVI devices.

\section{CONCLUSION}

There was a higher incidence of silent microinfarction with a greater number of lesions per patient following second-generation Lotus compared with the first-generation CoreValve implantation. However, there was no objective decline in neurocognitive function discernible at 1 year following TAVI with either prosthesis.

\section{Author affiliations}

${ }^{1}$ Multidisciplinary Cardiovascular Research Centre \& The Division of Biomedical Imaging, Leeds Institute for Cardiovascular and Metabolic Medicine, University of Leeds, Leeds, UK

${ }^{2}$ Cognitive Science Research Unit, University of Westminster, London, UK ${ }^{3}$ Interventional Cardiology, Leeds Teaching Hospitals NHS Trust, Leeds General Infirmary, Leeds, UK

${ }^{4}$ Department of Cardiovascular Sciences, BHF Cardiovascular Research Centre, University of Leicester, Leicester, UK

${ }^{5}$ National Institute of Health Research (NIHR) Cardiovascular Biomedical Research Unit, Glenfield Hospital, Leicester, UK

Contributors JPG conceived and designed the study. TAM drafted the manuscript. $\mathrm{CL}$ compiled the comprehensive battery of neurocognitive tests and instructed on their correct implementation. FR, TAM, AU, LED and AS performed the recruitment of patients and their neurocognitive assessment. GPM supervised and AS oversaw the University of Leicester contribution. MI and AJPG performed MRI image analysis. DJB and CM carried out TAVI implantation. TAM and AU analysed data and interpreted the results. PPS and PG gave input into data interpretation. PPS, PG, JRJF, GJF, SP and GPM were involved in critical and intellectual revision of the article. All authors edited and revised the manuscript. All authors read and approved the final manuscript.

Funding This study was part-funded by the British Heart Foundation (PG/11/126/29321), an investigator research grant from Boston Scientific and also the National Institute for Health Research (NIHR) Leeds Clinical Research Facility.

Competing interests DJB and CM are consultants and proctors for both Medtronic and Boston Scientific. JPG and SP have received an educational research grant from Philips Healthcare.

Patient consent for publication Not required.

Ethics approval National Research Ethics Service (08/H1307/106).

Provenance and peer review Not commissioned; externally peer reviewed.

Data sharing statement All clinically important data from this observational study have been reported on in the manuscript.

Open access This is an open access article distributed in accordance with the Creative Commons Attribution Non Commercial (CC BY-NC 4.0) license, which 
permits others to distribute, remix, adapt, build upon this work non-commercially, and license their derivative works on different terms, provided the original work is properly cited, appropriate credit is given, any changes made indicated, and the use is non-commercial. See: http://creativecommons.org/licenses/by-nc/4.0/.

\section{REFERENCES}

1. Vahanian A, Alfieri O, Andreotti F, et al. Guidelines on the management of valvular heart disease (version 2012). Eur Heart J 2012;33:2451-96.

2. Nishimura RA, Otto CM, Bonow RO, et al. 2014 AHA/ACC guideline for the management of patients with valvular heart disease: executive summary: a report of the American College of Cardiology/American Heart Association Task Force on Practice Guidelines. J Am Coll Cardiol 2014;63:2438-88.

3. Fairbairn TA, Meads DM, Hulme C, et al. The cost-effectiveness of transcatheter aortic valve implantation versus surgical aortic valve replacement in patients with severe aortic stenosis at high operative risk. Heart 2013;99:914-20.

4. Fairbairn TA, Meads DM, Mather AN, et al. Serial change in healthrelated quality of life over 1 year after transcatheter aortic valve implantation: predictors of health outcomes. J Am Coll Cardiol 2012;59:1672-80.

5. Leon MB, Smith CR, Mack MJ, et al. Transcatheter or Surgical Aortic-Valve Replacement in Intermediate-Risk Patients. N Engl J Med Overseas Ed 2016;374:1609-20.

6. Leon MB, Smith CR, Mack M, et al. Transcatheter aortic-valve implantation for aortic stenosis in patients who cannot undergo surgery. N Engl J Med 2010;363:1597-607.

7. Fearon WF, Kodali S, Doshi D, et al. Outcomes after transfemoral transcatheter aortic valve replacement: a comparison of the randomized PARTNER (Placement of AoRTic TraNscathetER Valves) trial with the NRCA (Nonrandomized Continued Access) registry. JACC Cardiovasc Interv 2014;7:1245-51.

8. Kahlert P, Knipp SC, Schlamann M, et al. Silent and apparent cerebral ischemia after percutaneous transfemoral aortic valve implantation: a diffusion-weighted magnetic resonance imaging study. Circulation 2010;121:870-8.

9. Ghanem A, Müller A, Nähle CP, et al. Risk and fate of cerebral embolism after transfemoral aortic valve implantation: a prospective pilot study with diffusion-weighted magnetic resonance imaging. $J$ Am Coll Cardiol 2010;55:1427-32.

10. Fairbairn TA, Mather AN, Bijsterveld P, et al. Diffusion-weighted MRI determined cerebral embolic infarction following transcatheter aortic valve implantation: assessment of predictive risk factors and the relationship to subsequent health status. Heart 2012;98:18-23.

11. Uddin A, Fairbairn TA, Djoukhader IK, et al. Consequence of cerebral embolism after transcatheter aortic valve implantation compared with contemporary surgical aortic valve replacement: effect on healthrelated quality of life. Circ Cardiovasc Interv 2015;8:e001913.

12. Vermeer SE, Prins ND, den Heijer T, et al. Silent brain infarcts and the risk of dementia and cognitive decline. N Engl J Med 2003;348:1215-22.

13. Meller SM, Baumbach A, Brickman AM, et al. Clinical implications for diffusion-weighted MRI brain lesions associated with transcatheter aortic valve replacement. Catheter Cardiovasc Interv 2014;83:502-8.

14. Meredith IT, Walters DL, Dumonteil N, et al. 1-Year outcomes with the fully repositionable and retrievable lotus transcatheter aortic replacement valve in 120 high-risk surgical patients with severe aortic stenosis: results of the REPRISE II Study. JACC CardiovasC Interv 2016:9:376-84.

15. Gooley RP, Talman AH, Cameron JD, et al. Comparison of selfexpanding and mechanically expanded transcatheter aortic valve prostheses. JACC Cardiovasc Interv 2015;8:962-71.

16. Musa TA, Uddin A, Dobson LE, et al. Cardiovascular magnetic resonance assessment of 1st generation CoreValve and 2nd generation Lotus valves. J Interv Cardiol 2018;31:391-9.
17. Vahanian A, Alfieri O, Al-Attar N, et al. Transcatheter valve implantation for patients with aortic stenosis: a position statement from the European Association of Cardio-Thoracic Surgery (EACTS) and the European Society of Cardiology (ESC), in collaboration with the European Association of Percutaneous Cardiovascular Interventions (EAPCI). Eur Heart J 2008;29:1463-70.

18. Piazza N, Grube E, Gerckens U, et al. Procedural and 30-day outcomes following transcatheter aortic valve implantation using the third generation (18 Fr) corevalve revalving system: results from the multicentre, expanded evaluation registry 1-year following CE mark approval. Eurolntervention 2008;4:242-9.

19. Meredith IT, Hood KL, Haratani N, et al. Boston Scientific Lotus valve. Eurolntervention 2012;8(Suppl Q):Q70-Q74.

20. Kappetein AP, Head SJ, Généreux P, et al. Updated standardized endpoint definitions for transcatheter aortic valve implantation: the Valve Academic Research Consortium-2 consensus document. Eur Heart J 2012;33:2403-18.

21. Hachinski V, ladecola C, Petersen RC, et al. National Institute of Neurological Disorders and Stroke-Canadian Stroke Network vascular cognitive impairment harmonization standards. Stroke 2006;37:2220-41.

22. Knipp SC, Kahlert P, Jokisch D, et al. Cognitive function after transapical aortic valve implantation: a single-centre study with 3-month follow-up. Interact Cardiovasc Thorac Surg 2013;16:116-22.

23. Ghanem A, Kocurek J, Sinning JM, et al. Cognitive trajectory after transcatheter aortic valve implantation. Circ Cardiovasc Interv 2013;6:615-24.

24. Zamvar V, Williams D, Hall J, et al. Assessment of neurocognitive impairment after off-pump and on-pump techniques for coronary artery bypass graft surgery: prospective randomised controlled trial. BMJ 2002;325:1268.

25. Crum RM, Anthony JC, Bassett SS, et al. Population-based norms for the Mini-Mental State Examination by age and educational level. JAMA 1993;269:2386-91.

26. Roach GW, Kanchuger M, Mangano CM, et al. Adverse cerebral outcomes after coronary bypass surgery. Multicenter Study of Perioperative Ischemia Research Group and the Ischemia Research and Education Foundation Investigators. N Engl J Med 1996;335:1857-63.

27. Kahlert P, Al-Rashid F, Döttger P, et al. Cerebral embolization during transcatheter aortic valve implantation: a transcranial Doppler study. Circulation 2012;126:1245-55.

28. Van Mieghem NM, El Faquir N, Rahhab Z, et al. Incidence and predictors of debris embolizing to the brain during transcatheter aortic valve implantation. JACC Cardiovasc Interv 2015;8:718-24.

29. Ringer TM, Neumann-Haefelin T, Sobel RA, et al. Reversal of early diffusion-weighted magnetic resonance imaging abnormalities does not necessarily reflect tissue salvage in experimental cerebral ischemia. Stroke 2001;32:2362-9.

30. Sacco RL, Kasner SE, Broderick JP, et al. An updated definition of stroke for the 21st century: a statement for healthcare professionals from the American Heart Association/American Stroke Association. Stroke 2013;44:2064-89.

31. Rodés-Cabau J, Dumont E, Boone RH, et al. Cerebral embolism following transcatheter aortic valve implantation: comparison of transfemoral and transapical approaches. J Am Coll Cardiol 2011;57:18-28.

32. Leon MB, Smith CR, Mack MJ, et al. Transcatheter or surgical aortic-valve replacement in intermediate-risk patients. $N$ Engl J Med 2016;374:1609-20.

33. Haussig S, Mangner N, Dwyer MG, et al. Effect of a cerebral protection device on brain lesions following transcatheter aortic valve implantation in patients with severe aortic stenosis: the CLEAN-TAVI Randomized Clinical Trial. JAMA 2016;316:592-601.

34. Kapadia SR, Kodali S, Makkar R, et al. Protection against cerebral embolism during transcatheter aortic valve replacement. J Am Coll Cardiol 2017;69:367-77.

35. Windham BG, Deere B, Griswold ME, et al. Small brain lesions and incident stroke and mortality: a cohort study. Ann Intern Med 2015;163:22-31. 